\title{
Measurement of Motion Error of Linear Stage
}

\author{
Ryoshu Furutani*, Jun Izawa and Miyu Ozaki \\ Tokyo Denki University Senju-Asahi 5, Adachi, 120-8551 TOKYO, Japan \\ ${ }^{*}$ Corresponding author
}

\begin{abstract}
In this paper, we propose measurement system of the six degree of motion errors which is based on distance measurement by the laser interferometer. The system has six parallel laser beams and six corner cube mirrors on the linear stage, which reflect the corresponding laser beams. The error of axial direction is measured with the ordinary distance measurement method by laser interferometer. The vertical errors to the axial direction and the roll errors are measured by tilted beams using the wedge prism. The pitch and yaw errors are measured by the difference between distance of two corner cube mirrors. The experimental layout of the corner cube mirrors and the other optical devices are shown. As a result, the resolution of $66.5 \mathrm{~nm}$ for error of the axial direction, the resolution of $383 \mathrm{~nm}$ for the vertical errors to the axial direction, the resolution of 1.23 arc-sec for the roll error and the resolution of 0.429 arc-sec for the pitch and yaw errors are obtained in this system.
\end{abstract}

Keywords-component; measurement of kinematic errors; laser interferometer; displacement measurement; angle measurement

\section{INTRODUCTION}

Recently, in order to the improve the accuracy of optical devices and semiconductor wafers, the improvement of positioning accuracy of a machine tool table and a coordinate measuring machine (CMM) carriage have been required. For the purpose of increasing the positioning accuracy, geometric errors of the table determined from vertical errors to the feed direction of a table and angle errors around each axis should be measured and calibrated. These errors are called the geometric errors. If some or all of these geometric errors can be measured simultaneously, because influence of environmental noise such as variation of temperature and vibration can be decreased, high accurate compensation could be expected. However, usually there is not enough space around a machine tool to set up the whole measurement instruments such as displacement sensors and autocollimators. So it is difficult to simultaneous measure of six degree of motion errors with the conventional measuring instruments.

Reference [1] provided the multi-degree-of-freedom measurement (MDFM) system for CMM. The MDFM system can measure five errors except positioning error along axial direction by using four lasers and position sensitive detectors (PSDs). The other studies were employed laser interferometer in regards to positioning and laser beams position on PSDs or quadrant photo detectors (QPDs) in regards to other errors [24]. [5] employed laser Doppler displacement meter for positioning and the interferometric principle for pitch and yaw. Reference [6] also measured six motion errors of $x-y$ stage using four Laser Doppler Scale for $\mathrm{x}, \mathrm{y}$, pitch and yaw and two QPDs for vertical error and roll.
In this paper, the measurement system that employs principle of laser interferometer for all motion errors is proposed. High resolution should be expected, if the system employs a commercial laser interferometer.

\section{MEASUREMENT PRINCIPLE}

\section{A. Layout of the System}

The schematic diagram of measurement system is shown in Figure I. The corner cube mirrors CC1 CC6 fixed on the bracket are movable and the other parts are fixed as similar as Michelson type interferometer. The unit of corner cube mirrors is set on a table of a machine tool in actual situation.

The laser beam is divided into six laser beams. Six laser beams are collimated by collimate lens CL. Six laser beams are split by beam splitter $\mathrm{BS}$ into laser beams heading for reference mirror $\mathrm{RM}$ and corner cube mirrors $\mathrm{CC}_{1} \sim \mathrm{CC}_{6}$. The laser beams heading for RM enter and are reflected by RM. And then, the laser beams pass through BS and enter photo detectors PDs. Three beams out of six laser beams heading for $\mathrm{CC}_{1} \sim \mathrm{CC}_{6}$ are tilted by wedge prisms WPs that are installed between $\mathrm{BS}$ and $\mathrm{CC}_{1} \sim \mathrm{CC}_{6}$ to measure the vertical errors to the axial direction $\Delta y$ and $\Delta z$, and the roll errors $\Delta \theta_{\mathrm{x}}$. Six laser beams are reflected by $\mathrm{CC}_{1} \sim \mathrm{CC}_{6}$ and vertically enter the plane mirrors PM. To return the laser beams to original pass, PMs are installed between WPs and $\mathrm{CC}_{1} \sim \mathrm{CC}_{6}$. The laser beams are reflected by $\mathrm{PMs}$ and enter and are reflected by $\mathrm{CC}_{1} \sim \mathrm{CC}_{6}$ again. And then, the lasers are reflected by BS and enter PDs. The reflected lasers from both mirrors finally interfere with each other. Displacement of the target mirror can be measured by counting interference fringes of brightness and darkness of the interference laser beam.

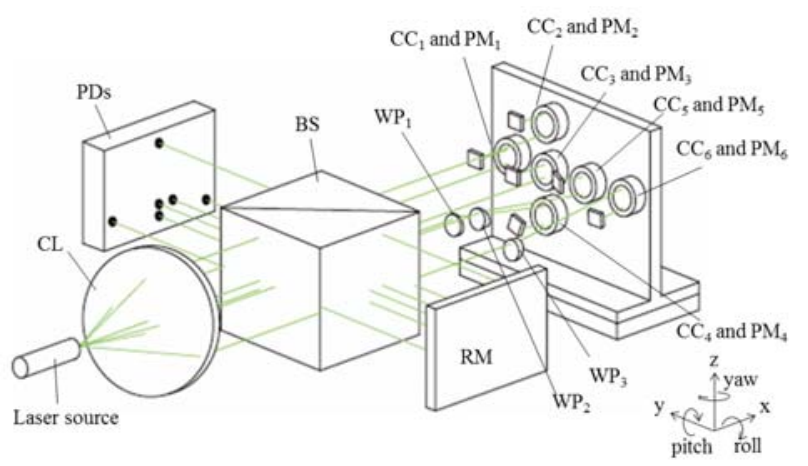

FIGURE I. MEASUREMENT PRINCIPILE OF THE VERTICAL ERROR $\Delta \mathrm{y}$ 
B. Measurement Principle of the Displacement of Axial Direction $\Delta x$

To measure displacement $\Delta \mathrm{x}, \mathrm{CC} 3$ is used. Relations between displacement $\Delta \mathrm{x}$ and number of fringes is shown in (1).

$$
\Delta \mathrm{x}=\mathrm{n} \lambda / 4
$$

where $\mathrm{n}$ denotes number of interference fringes and $\lambda$ denotes wave length of the laser beam.

\section{Measurement Principle of the Vertical Errors to the Axial Direction $\Delta y$ and $\Delta z$}

To measure the vertical error $\Delta \mathrm{y}, \mathrm{WP}_{2}, \mathrm{CC}_{5}$ and $\mathrm{PM}_{5}$ are used. The schematic diagram for $\Delta y$ measurement is shown in Figure II. Layout of $\mathrm{WP}_{1}, \mathrm{CC}_{4}$ and $\mathrm{PM}_{4}$ for vertical error $\Delta \mathrm{z}$ measurement is just to make the same layout for $\Delta y$ measurement 90-degree turn around axial direction. When the $\mathrm{CC}_{5}$ or $\mathrm{CC}_{4}$ moves along vertical direction, the vertical errors $\Delta y$ and $\Delta z$ can be obtained in (2) and (3).

$$
\begin{aligned}
& \Delta y=\mathrm{n} \lambda /(4 \sin \theta) \\
& \Delta \mathrm{z}=\mathrm{n} \lambda /(4 \sin \theta)
\end{aligned}
$$

where $\mathrm{n}$ denotes number of interference fringes, $\lambda$ denotes wave length of the laser beam, and $\theta$ denotes the angle given by the wedge prism.

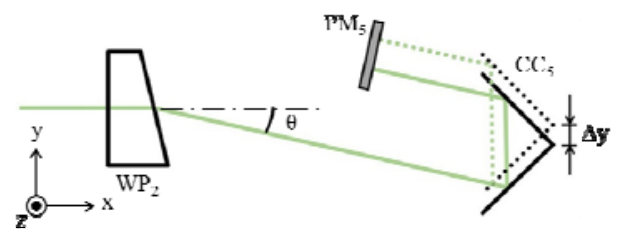

FIGURE II. MEASUREMENT PRINCIPLE OF THE VERTICAL ERROR $\Delta \mathrm{y}$

\section{Measurement Principle of Angle Error Roll}

To measure the angle error roll, $\mathrm{WP}_{3}$ and $\mathrm{CC}_{6}$ are used. The schematic diagram for roll measurement is shown in Figure III. In our layout, the center of rotation is designed as the center of $\mathrm{CC}_{3}$. When the roll is small, $\mathrm{CC}_{6}$ moves along $\mathrm{z}$ direction. The moving distance is measured as the same way for measuring $\Delta \mathrm{z}$ we showed in 2.C. From this $\mathrm{z}$ direction displacement and distance between center of rotation and $\mathrm{CC}_{6}$, roll $\Delta \theta_{\mathrm{x}}$ can be measured and can be obtained from (4).

$$
\Delta \theta_{\mathrm{x}}=\tan { }^{-1}\left(\mathrm{n} \lambda / 4 \mathrm{~L}_{36} \sin \theta\right)
$$

where $\mathrm{n}$ denotes number of interference fringes, $\lambda$ denotes wave length of the laser beam, $\theta$ denotes the angle given by the wedge prism, and $\mathrm{L}_{36}$ denotes distance between center of $\mathrm{CC}_{3}$ and center of $\mathrm{CC}_{6}$.

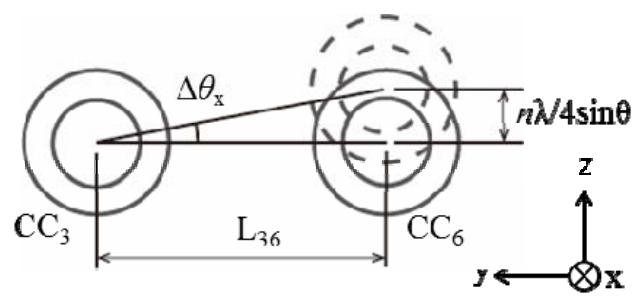

FIGURE III. MEASUREMENT PRINCIPLE OF THE ANGLE ERROR $\Delta \theta_{\mathrm{x}}$

\section{E. Measurement Principle of Angle Errors Pitch and Yaw}

To measure the angle error pitch, $\mathrm{CC}_{2}$ is used. The schematic diagram for pitch measurement is shown in Figure IV. Layout of $\mathrm{CC}_{1}$ for the angle error yaw $\Delta \theta_{z}$ measurement is just to make the same layout for pitch measurement 90 -degree turn around axial direction. When the pitch error is caused, $\mathrm{CC}_{2}$ moves along $\mathrm{x}$ direction. From this $\mathrm{x}$ direction displacement and distance between center of rotation and $\mathrm{CC}_{2}$, pitch $\Delta \theta_{\mathrm{y}}$ can be measured and can be obtained from (5) and yaw $\Delta \theta_{z}$ similarly can be obtained from (6).

$$
\Delta \theta_{\mathrm{y}}=\tan ^{-1}(\mathrm{n} \lambda / 4 \mathrm{~L})
$$

$$
\Delta \theta_{\mathrm{z}}=\tan ^{-1}(\mathrm{n} \lambda / 4 \mathrm{~L})
$$

where $\mathrm{n}$ denotes number of interference fringes, $\lambda$ denotes wave length of the laser beam, and $\mathrm{L}$ denotes distance between center of rotation and $\mathrm{CC}_{2}$ or $\mathrm{CC}_{1}$.

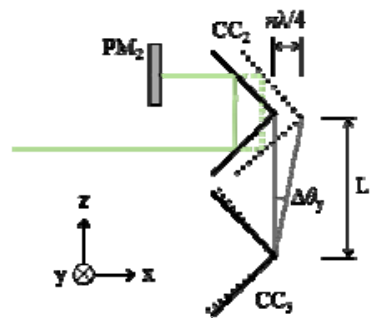

FIGURE IV. MEASUREMENT PRINCIPLE OF THE ANGLE ERROR $\Delta \theta_{\mathrm{y}}$

\section{EVALUATION EXPERIMENT}

To evaluate the proposed system, some moving value were given to translation, rotation and gonio stages respectively and each displacements were measured in the system. Measured values obtained from the system are compared with moving value of stages. The used laser wave length is $532 \mathrm{~nm}$. The wedge prisms that give the laser beams 10 degree were used, so that $\theta$ in (2), (3) and (4) are 10 degree. The differences of each corner cube distance were designed $32 \mathrm{~mm}$, so that $\mathrm{L}$ in (5) and (6) are $32 \mathrm{~mm}$ and L36 in (4) is $64 \mathrm{~mm}$.

Stages are given certain moving values along each axial direction and are returned to initial position after measuring once. For easy to reading the number of times of sine curve 
periods of the interference fringes, the stages are given increase feeds of every $0.133 \mu \mathrm{m}$ for $\mathrm{x}$ direction, $0.766 \mu \mathrm{m}$ for $\mathrm{y}$ and $\mathrm{z}$ direction, 0.00478 degree for roll and 0.00476 degree for pitch and yaw. Sine curves of the interference fringes are read in increments of 0.5 times periods. Accordingly, reading errors of $0.133 \mu \mathrm{m}$ in $\Delta \mathrm{x}, 0.766 \mu \mathrm{m}$ in $\Delta \mathrm{y}$ and $\Delta \mathrm{z}, 0.000686$ degree in $\Delta \theta_{\mathrm{x}}, 0.000238$ degree in $\Delta \theta_{\mathrm{y}}$ and $\Delta \theta_{\mathrm{z}}$ exists. Measured values are regarded as two digits significant figures due to reading errors.

Errors of the measured values obtained from the system are shown in Figure V. Each lines denotes the mean value of measured values of each directions. Standard deviations 0.062 $\mu \mathrm{m}$ for $\mathrm{x}, 0.28 \mu \mathrm{m}$ for $\mathrm{y}$ and $0.028 \mu \mathrm{m}$ for $\mathrm{z}$ are obtained in the range of about $10 \mu \mathrm{m}$ travel of each directions and 0.0013 degree for roll, 0.00092 degree for pitch and 0.00095 degree for yaw were obtained in the range of about \pm 0.05 degree of each rotational angles. A series of increases errors can be found in roll error. It was because the small displacements ignored as reading errors accumulated in proportion to increases of movement amounts of the stage and appeared as displacements. It is assumed that small displacement was caused by maladjustment of the system. Also, $\Delta y$ are especially affected by maladjustment of $\mathrm{WP}_{2}$. Decrease of $\Delta y$ at $5 \mu \mathrm{m}$ stage travel are assumed to be caused by the reading errors.

\section{CONCLUSION}

The measurement system that employs principle of laser interferometer for all motion errors of stages were proposed. To evaluate the system, measured value were compared with the displacements of each directions and rotation angles given to the translation, rotation and gonio stages. Standard deviations $0.062 \mu \mathrm{m}$ for $\mathrm{x}, 0.28 \mu \mathrm{m}$ for $\mathrm{y}$ and $0.028 \mu \mathrm{m}$ for $\mathrm{z}$ are obtained in the range of about $10 \mu \mathrm{m}$ travel of each directions and 0.0013 degree for roll, 0.00092 degree for pitch and 0.00095 degree for yaw were obtained in the range of about \pm 0.05 degree of each rotational angles. If the system employs a commercial laser interferometer, high accuracy are expected because reading errors could be decreasing. Roll error were measured by designing center of the system to conform to the center of rotation of the gonio stage. Roll measurement system not depending on center of rotation could be done to install one more system as same as proposed roll measurement system because roll angle can be calculated from differences of output of two systems.

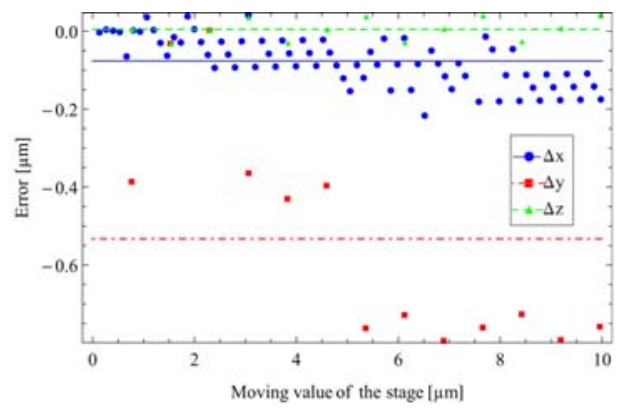

(a) Results of translation motion

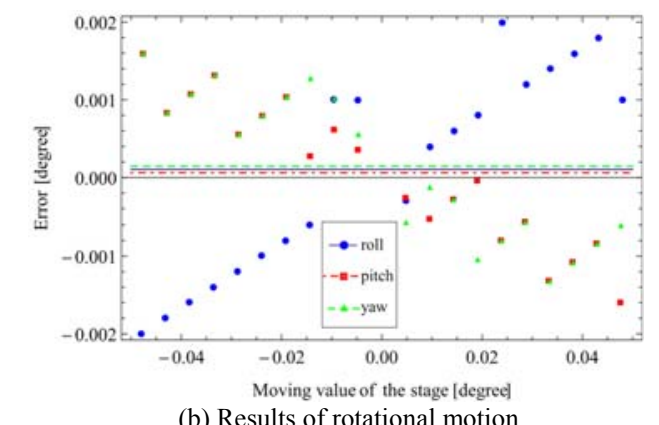

(b) Results of rotational motion

FIGURE V. RESULTS OF EVALUATION MEASUREMENT

\section{ACKNOWLEDGMENT}

This work was partially supported by Research Institute for Science and Technology of Tokyo Denki University Grant Number Q15T02 / Japan.

\section{REFERENCES}

[1] Ni, J., Huang, P. S. and Wu, S. M., A Multi-Degree-of-Freedom Measuring System for CMM Geometric Errors, Journal of Engineering for Industry, Transactions of the ASME, 1992, 114, 362-369.

[2] Q. Feng, B. Zhang, C. Cui, C. Kuang, Y. Zhai and F. You, Development of a simple system for simultaneously measuring 6DOF geometric motion errors of a linear guide, Optics Express, 2013, 21, 22, 2580525819

[3] Shimizu, S., Lee, H. -S. and Imai, N, Simultaneous measuring method of table motion errors in 6 degree of freedom, Int. J. Japan Soc. Prec Eng, 1994, 28, 3, 273-274.

[4] C.H. Liu, W. Y. Jywe, C. C. Hsu and T. H. Hsu, Development of a laserbased high-precision six-degrees-of freedom motion errors measuring system for linear stage, REVIEW OF SCIENTIFIC INSTRUMENT, 2005, 76, 055110

[5] Q. Feng, B. Zhang, C. Cui, C. Kuang, Y. Zhai and F. You, Development of a simple system for simultaneously measuring 6DOF geometric motion errors of a linear guide, Optics Express, 2013, 21, 22, 2580525819

[6] Fan KC, Chen MJ, Huang WM, A six-degree-of-freedom measurement system for the motion accuracy of linear stages, International Journal of Machine Tools and Manufacture, 1998, 38: 155-164, 1979.

[7] Fan KC, Chen MJ, A 6-degree-of-freedom measurement system for the accuracy of X-Y stages, Precision Engineering, 2000, 24, 15-23 